\title{
The Association Between Hyperparathyroidism and Ischemic Stroke Subtypes
}

\author{
Halil Onder ${ }^{\mathrm{a}, \mathrm{b}}$, Guven Arslan ${ }^{\mathrm{a}}$
}

\begin{abstract}
Background: Up to date, a substantial amount of research has remarked on the potential role of parathormone (PTH) in the development of subclinical and clinical vascular diseases. However, the association between the hyperparathyroidism and cerebrovascular disease has rather been underestimated in the literature. Herein, we aimed to investigate the association between serum PTH levels and ischemic stroke.
\end{abstract}

Methods: Serum PTH levels were measured in all patients with ischemic stroke who were hospitalized in the Yozgat City Hospital between January 1, 2017 and January 1, 2019. Clinical and demographic findings were retrospectively evaluated via computer-based patient record system of Yozgat City Hospital (AKGUN).

Results: Overall, 158 patients with ischemic stroke with a median age of $71.5 \pm 11.5$ were enrolled in this study. Parathyroid hormone was found to be high in 31 of the patients $(19.6 \%)$. The stroke subtype of extracranial atherosclerosis was found to be more common in the group of patients with a high level of PTH $(12 \% / 3 \% ; P=0.008)$. Remarkably, logistic regression analyses also confirmed that high PTH level was a significant variable in the determination of the stroke subtype of extracranial atherosclerosis $(\mathrm{P}=0.024)$.

Conclusions: We have found a high rate of hyperparathyroidism in our group of patients with ischemic stroke. Remarkably, the elevation of PTH was found to be significantly associated with the ischemic stroke subtype of extracranial atherosclerosis. Clarification of these results in the future large-scale studies may provide crucial perspectives regarding our understanding of the pathophysiology of some subtypes of ischemic stroke and potentially lead to a large public health implication in this area.

Keywords: Parathormone; Stroke; Risk factor; Screening; Pathophysiology

Manuscript submitted December 31, 2019, accepted January 21, 2020

${ }^{a}$ Neurology Clinic, Yozgat City Hospital, Yozgat, Turkey

${ }^{\mathrm{b}}$ Corresponding Author: Halil Onder, Neurology Clinic, Yozgat City Hospital, Yozgat, Turkey. Email: halilnder@yahoo.com

doi: https://doi.org/10.14740/jnr564

\section{Introduction}

There is a substantial amount of research suggesting that parathormone (PTH) may be involved in the development of subclinical and clinical vascular diseases through mechanisms of endothelial dysfunction, increased vascular stiffness, hypertension, and atherosclerosis [1-3]. However, although its association with cardiovascular diseases and increased risk of atherosclerosis have been investigated frequently, the number of studies particularly focusing on the association between PTH elevation and stroke [4-6], and cerebral atherosclerosis is quite limited [2]. In a crucial study by Sato et al, increased PTH levels were found in female patients with ischemic stroke, and the authors drew attention to the possible relation between hyperparathyroidism and stroke [5]. More recently, Celik et al remarked on serum PTH level as a potential predictor for determination of stroke risk [6]. They also emphasized the need for future studies to investigate the effect of PTH on stroke risk. The determination of the potential importance of the PTH elevation as a risk factor for stroke would certainly contribute substantially to our understanding of the pathophysiology of the unknown aspects of stroke and potentially lead to a large public health implication in this area. Taken together, we aimed to reinvestigate the frequency of PTH elevation in our cohort of patients with ischemic stroke. Remarkably, we focused on its relationship with specific stroke subtypes which was not examined previously.

\section{Materials and Methods}

In this prospective study, all patients with ischemic stroke who were hospitalized in the Yozgat City Hospital between January 1, 2017 and January 1, 2019 were evaluated $(\mathrm{n}=165)$. Patients without a demonstratable acute ischemic lesion on diffusion magnetic resonance imaging (MRI) were excluded from the analyses $(\mathrm{n}=7)$. The data of the remaining 158 patients were included in the study analyses. Blood samples for analyses of PTH were obtained immediately after the stroke. Serum PTH levels were measured using a chemilu-minescence method in a DXI 800 Access Immunoassay System analyzer (Beckman Coulter Inc., Brea, CA, USA) and the reference range was set at $10-80 \mathrm{pg} / \mathrm{mL}$. Clinical and demographic findings were retrospectively evaluated via Computer-Based Patient Record System of Yozgat City Hospital (AKGUN). In our center, all the patients with ischemic stroke are routinely investigated 
with paraclinical methods including echocardiography, electrocardiography, 24-h Holter electrocardiogram (ECG) monitoring, and a vascular imaging method (computed tomography (CT) angiography of the neck and brain). The results of the etiological investigations and particularly vascular imaging were examined and classification of the stenosis of large vessels was performed as: $50 \%,>50-70 \%$, and $>70 \%$. Classification of the subtypes of ischemic stroke was made according to the trial of ORG 10172 in Acute Stroke Treatment (TOAST) classification [7]. The study was approved by the local ethics committee of Bozok Medical School, and it was conducted in accordance with the Declaration of Helsinki.

\section{MRI protocol}

All MRI scans were performed on a Siemens 1.5 Tesla Magnetom Amira MRI scanner using 5-mm slice thickness and apparent diffusion coefficient (ADC).

\section{Statistical analysis}

Descriptive statistics on SPSS 20 were used to analyze the clinical demographical features of the overall patients. Chi-square statistics were used to compare the clinical and demographic features of the patients with high PTH and normal PTH levels. Mann-Whitney $U$ test was performed to compare the serum PTH levels between female and male genders. Binary logistic regression analyses were used to investigate the predictor values of multiples factors in the determination of stroke subtypes. In addition to PTH status, a model taking into consideration the known risk factors for extracranial atherosclerosis was formed. $\mathrm{P}$ values and odds ratios for all the variables have been calculated in the analyses.

\section{Results}

Overall, 158 patients with ischemic stroke were enrolled in this study. Female/male ratio was 79/79 and the mean age of the overall group was $71.5 \pm 11.5(31-96)$. The interrogation of the clinical data for risk factors of stroke revealed that comorbidity of hypertension was present in $130(82.3 \%)$ of the patients. Other risk factors including hyperlipidemia, diabetes mellitus, myocardial infarction, coronary artery disease and atrial fibrillation were found to be present at ratios of $34.2 \%$ $(\mathrm{n}=54), 35.4 \%(\mathrm{n}=56), 2.5 \%(\mathrm{n}=4), 22.8 \%(\mathrm{n}=36), 27.2 \%$ $(\mathrm{n}=43)$, respectively (Table 1$)$. Serum PTH hormone values were found to be high in 31 of the patients $(19.6 \%)$. The mean level of PTH in the overall group was $67.7 \pm 42.5 \mathrm{pg} / \mathrm{mL}$. The mean level of PTH in the high PTH group was $135.2 \pm 48.8$ $\mathrm{pg} / \mathrm{mL}$, whereas it was $51.2 \pm 17.2 \mathrm{pg} / \mathrm{mL}$ in the group with normal level of PTH. Based on a previous report remarking on the gender difference of stroke patients in terms of PTH level, we also compared the hyperparathyroidism frequency and PTH levels between gender groups. Chi-square analyses did not reveal a significant difference in terms of PTH status (high
Table 1. Demographic and Clinical Features of the Study Group With Ischemic Stroke $(n=158)$

\begin{tabular}{ll}
\hline & Mean \pm SD \\
\hline Age, year & $71.5 \pm 11.5$ \\
Gender (female/male) & $79 / 79$ \\
\hline Parathormone, pg/mL & $67.7 \pm 42.5$ \\
\hline & N (\%) \\
\hline Large vessel imaging & \\
\hline Normal large vessel & $131(82.9 \%)$ \\
\hline Stenosis $>50 \%$ & $13(8.2 \%)$ \\
\hline Stenosis $>70 \%$ & $14(8.9 \%)$ \\
\hline Stroke classification & \\
\hline LAA (intracranial) & $56(35.4 \%)$ \\
\hline LAA (extracranial) & $8(5 \%)$ \\
\hline Cardioembolism & $37(23.4 \%)$ \\
\hline Lacune & $52(32.9 \%)$ \\
Stroke of other determined etiology & $0(0 \%)$ \\
Stroke of undetermined etiology & $5(3.2 \%)$ \\
\hline Hypertension & $130(82.3 \%)$ \\
Hyperlipidemia & $54 / 158(34.2 \%)$ \\
Diabetes mellitus & $56 / 158(35.4)$ \\
Myocardial infarction & $4 / 158(2.5 \%)$ \\
Coronary artery disease & $36(22.8 \%)$ \\
\hline Atrial fibrillation & $43(27.2 \%)$ \\
\hline
\end{tabular}

F/M: female/male; SD: standard deviation; LAA: large-artery atherosclerosis. ${ }^{*} \mathrm{P}<0.05$.

or normal) between female and male genders (19/79 (24\%), $12 / 79(15 \%), P=0.22)$. However, when the values of PTH were compared between gender groups, it was found that the values were significantly higher in female stroke patients (the mean value was $75.3 \pm 49.2$, whereas it was in male group 60.1 $\pm 33.2, \mathrm{P}=0.023$, Man-Whitney U Test). Vascular imaging results revealed that extracranial artery stenosis of more than $50 \%$ was found in $8.2 \%(n=13)$ of the patients whereas stenosis more than $70 \%$ was found in $8.9 \%(n=14)$ of the patients. Fifty-one of the patients $(32.3 \%)$ were diagnosed with lacunar stroke, 64 of them $(40.6 \%)$ were diagnosed with large vessel atherosclerosis, and 37 of them $(23.4 \%)$ were diagnosed with cardioembolic stroke (Table 1).

The incidences of risk factors for stroke were compared in the group of patients with normal PTH values and high PTH values (Table 2). The results of the analyses revealed that extracranial atherosclerosis was found to be more common in the group of patients with high PTH levels $(12 \% / 3 \% ; \mathrm{P}=0.008$, Chi-square test). Logistic regression analyses also confirmed that elevated PTH was a significant variable in the determination of the stroke subtype of extracranial atherosclerosis $(\mathrm{P}=$ 0.024) (Table 3). However, there was no statistical difference in terms of PTH status (high or normal) when the classification 
Table 2. Comparisons of the Demographic and Clinical Features Between High PTH Group and Normal PTH Group of Patients

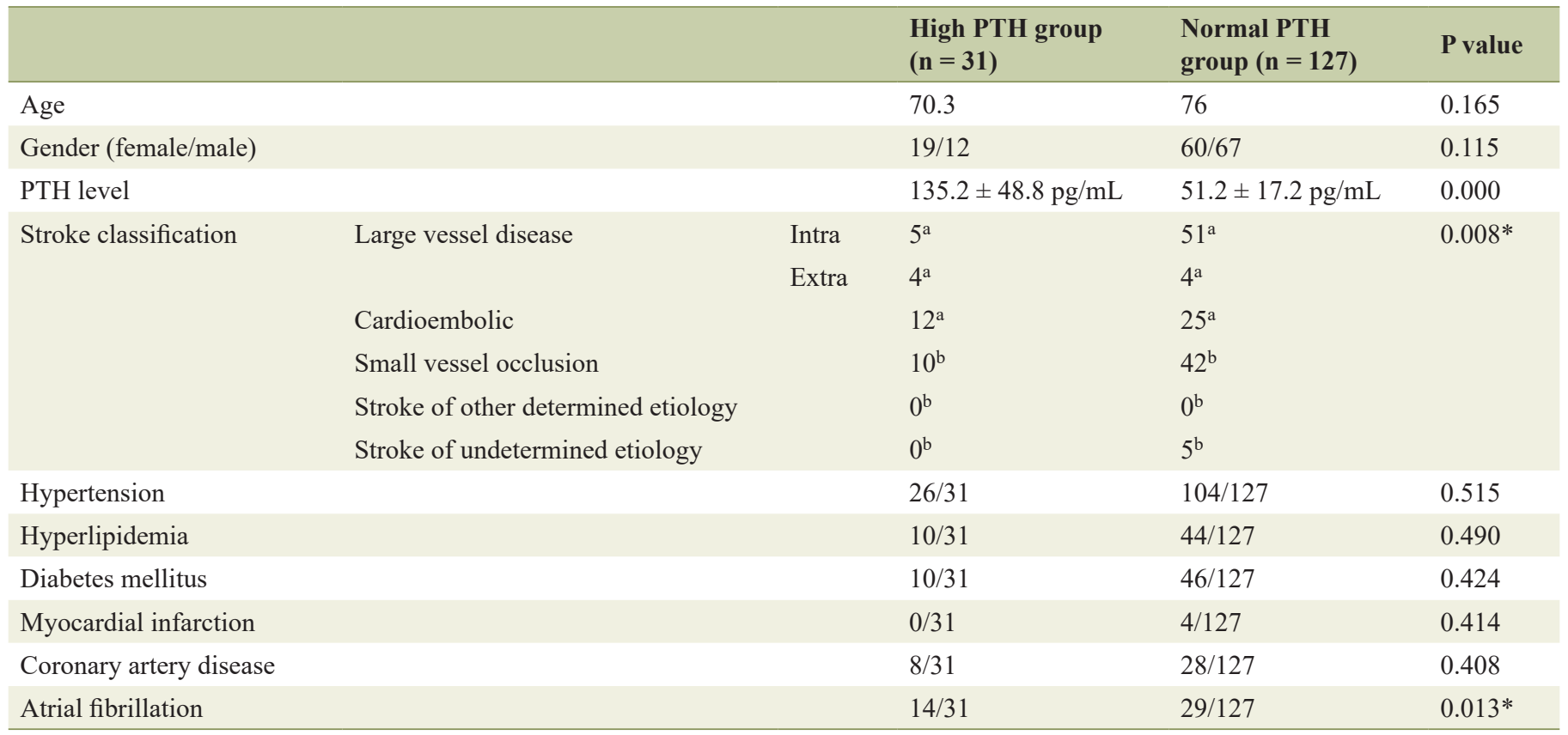

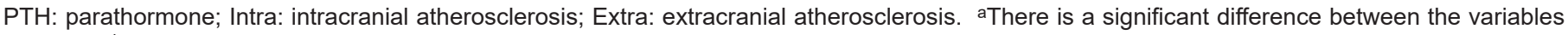

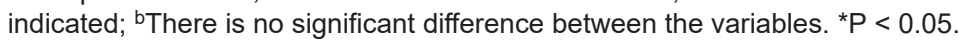

was performed as large vessel atherosclerosis (intracranial and extracranial atherosclerosis) and others $(\mathrm{P}=0.514)$. In a previous report, Sato et al had tried to explain the association between hyperparathyroidism and stroke via a mechanism of the increased incidence of hypertension in hyperparathyroidism [5]. However, we have not found a statistical difference in the frequency of hypertension between groups of patients with a normal and high level of PTH $(\mathrm{P}=0.515)$.

\section{Discussion}

Classically, primary hyperparathyroidism is accepted as a disease with increased cardiovascular morbidity and mortality [8]. In a recent study, the elevation of PTH was found to be useful as a reliable predictor of coronary artery calcification [9]. In the study including the largest cohort of patients, the association between PTH elevation and the atherosclerosis was investigated using whole-body magnetic resonance angiography, a novel way of assessing the total atherosclerotic burden in the vasculature outside the heart and brain [4]. In conclusion of their study, the authors found that PTH was associated both with the degree of atherosclerosis and risk of clinically overt atherosclerotic disease. They remarked on the need for future studies focusing on the utility of measuring PTH in a clinical setting for risk assessment of atherosclerotic disease [4]. However, there are also some researchers who have not confirmed this finding, such that patients with mild hyperparathyroidism were found to have reduced cardiovascular mortality (relative risk of 0.6) [10]. Besides, the causal relationship between hy-

Table 3. Multiple Logistic Analysis of Potential Risk Factors and Diseases Associated With Ischemic Stroke due to Extracranial Atherosclerosis and Other Subtypes

\begin{tabular}{|c|c|c|c|c|}
\hline Risk factors & Extracranial atherosclerosis $(n=8)$ & Other subtypes $(n=150)$ & Odds ratio $(95 \% \mathrm{CI})$ & P value \\
\hline Hypertension & $6 / 8$ & $124 / 150$ & $0.860(0.120-6.167)$ & 0.881 \\
\hline Hyperlipidemia & $4 / 8$ & $50 / 150$ & $2.819(0.573-13.876)$ & 0.202 \\
\hline Myocardial infarction & $0 / 8$ & $4 / 150$ & $5.440(0.000-0.000)$ & 1.000 \\
\hline Coronary artery disease & $0 / 8$ & $36 / 150$ & $0.000(0.000-0.000)$ & 0.998 \\
\hline PTH level (high/normal) & $4 / 4$ & $27 / 123$ & $0.164(0.034-0.788)$ & $0.024 *$ \\
\hline
\end{tabular}

Cl: confidence interval. * $\mathrm{P}<0.05$. 
perparathyroidism and vascular disease is unclear, such that if the PTH is the primary agent disturbing the vascular changes or another mediator such as serum calcium is the primarily responsible one [2]. On the other hand, as the early diagnosis and appropriate treatment rates have increased in the near future, clinical findings in hyperparathyroidism have become more subtle [2]. Hence, cardiovascular manifestations of the disease have become less common as well as researches focusing on this association have decreased in number and severity. In addition, although reports have generally focused on the association between PTH and cardiovascular disease, the association of PTH with cerebrovascular diseases has been investigated rather in a limited number $[5,11,12]$. Therefore, we think that the results of our study which are extremely rare in literature may give crucial perspectives regarding this interesting topic of the possible association between hyperparathyroidism and stroke. In our study, we have determined that an extremely high ratio of patients with ischemic stroke (19.6\%) had also hyperparathyroidism. In a previous report by Sato et al, onethird of the female patients with ischemic stroke were determined to have a PTH level higher than the mean +2 standard deviation (SD) of the control subjects [5]. In the other report by Celik et al including 60 patients with ischemic stroke and 79 healthy individuals, PTH levels together with 25-hydroxyvitamin $\mathrm{D}(25(\mathrm{OH}) \mathrm{D})$ were found to be as important factors in the determination of stroke risk [6]. Of note, in this study, the patients were not grouped as high PTH and normal PTH group. A significant limitation of our study was that we have not included control subjects to compare the prevalence of hyperparathyroidism between patients with ischemic stroke and healthy population. Although there is no prevalence study in our country, in a population-based study in the USA, the prevalence of primary hyperparathyroidism was estimated at $0.86 \%$ in 2008 - 2009 which may give a perspective while interpreting our results [13]. Distinctly, in an ancient study, Bostrom et al investigated the incidence of stroke in patients with hyperparathyroidism $(\mathrm{n}=170)$, which was determined as $7.1 \%$, a considerably high rate according to the incidence in the healthy population [4]. In conclusion of this study, the authors discussed the possible importance of normalization of the parathyroid function in these patients in order to facilitate the rehabilitation and prevent recurrence of stroke.

A crucial discussion may be the potential impact of gender in this association of PTH elevation and ischemic stroke. Remarkably, Sato et al concluded that high serum PTH level may be associated with the high incidence of ischemic stroke in women; however, they did not find a significant difference in PTH levels in male stroke patients in comparison to healthy subjects. They tried to explain these findings of distinct levels of PTH according to the gender in the setting of varying hormonal status in female stroke gender in the postmenopausal period [5]. In our analyses, we have also found that mean PTH levels were significantly higher in the female gender, although the results of the Chi-square tests did not reveal a significance. The significance of gender as an interplay in this association between hyperparathyroidism and ischemic stroke was not investigated in the above-referenced report by Celik et al [6]. We think that the possible effect of PTH and its varying significance according to the gender should certainly be investigated in a larger number of patients in the future reports.

A strictly crucial aspect of our study was that we have also specifically investigated the relationship between hyperparathyroidism and ischemic stroke subtypes which has not been investigated previously in detail. We think that our results in this regard are strictly remarkable in light of the very limited literature data. Currently, the association between hyperparathyroidism and stroke still needs to be clarified; however, the underlying pathophysiology of this possible association further remains obscure. Hyperparathyroidism has been shown to be frequently associated with hypertension [14]. Besides, Sato et al also found elevated serum PTH levels and hypertension prevalence at significantly higher rates in female stroke patients according to control subjects [5]. Combining these data, they hypothesized that the results of elevated PTH level in ischemic stroke patients may be related to an increased incidence of hypertension in patients with hyperparathyroidism. In a large-scale study by Hagstrom et al, PTH was found to predict clinically diagnosed vascular dementia as well as neuroimaging indices of cerebral small vessel disease (a subgroup including lacunar infarction) [15]. However, in this report, patients with acute stroke were not included in the study [15], which was methodologically distinct. In our study, we have not found a difference in the frequency of hypertension between the group of patients with high PTH and normal PTH levels. Hypertension is accepted as a risk factor for both lacunar infarcts [16-18] and large vessel atherosclerosis [19]. Nonetheless, its role in lacunar infarcts in terms of causal relationship has been shown more strongly such that Wang et al found that hypertension was specifically related to the recurrent strokes in patients with lacunar infarcts, not other subtypes of ischemic stroke syndromes [17]. Arrestingly, we have not found an association between high PTH level and lacunar stroke subtypes. On the other hand, a strong relationship between high PTH levels and extracranial atherosclerosis was determined in crosstabs analyses. We also confirmed these results in the multivariate logistic analyses when we excluded the interactions of other etiological causal agents for extracranial atherosclerosis [19] including hypertension, hyperlipidemia, diabetes mellitus, myocardial infarction, coronary artery disease, atrial fibrillation, and male gender $(\mathrm{P}=0.024$; odds ratio (95\% confidence interval (CI)) $0.164(0.034-0.788))$. These results were totally discordant with the study results of Sato et al, in which lacunar infarctions were found to be more prevalent in stroke patients of high PTH group. Lacunar infarctions and large vessel atherosclerosis are distinct entities in terms of clinical presentations, etiopathogenesis as well as classification [7]. For instance, whereas smoking and dyslipidemia are associated more strongly with large vessel atherosclerosis, diabetes mellitus has been shown to be more specific for lacunar infarction [20]. Regarding the pathophysiological aspects, lacunar infarctions develop due to abnormal tiny vessels often containing focal enlargements and small hemorrhagic extravasations [21]. Also, lipohyalinosis is accepted as a major cause of lacunar infarctions [22], whereas atherosclerosis is the main agent responsible for extracranial atherosclerosis [23]. Therefore, we think that the results of our study taken together with the conflicting results of the study by Sato et al should be interpreted cautiously. In our opinion, the major limitation of the study 
by Sato et al was that strokes were classified according to the Classification of Cerebrovascular Diseases (version III) of the US National Institute of Neurological Disorders and Stroke which was in the form of two subtypes, atherothrombotic and lacunar infarctions [5]. There was no an information regarding the prevalence of cardioembolic strokes and data regarding the large vessel imaging was not included in this study which might constitute the major limitations (furthermore, no group of extracranial atherosclerosis was compromised). In literature, the association between serum PTH hormone and atherosclerotic disease has been shown confidently in the previous studies $[3,24]$. In another case-control study by Walker et al, the authors found that carotid stiffness (a subclinical predictor of systemic atherosclerosis) was associated with the extent of PTH elevation and they concluded that impaired vascular compliance might be associated primarily with PTH elevation [2]. Taken together, we also think that the results of our study were in accordance with the related previous reports using distinct (indirect) methods above mentioned [2, 3]. However, future reports investigating the possible association between hyperparathyroidism and distinct subtypes of ischemic stroke are surely warranted to clarify these discussions. The studies may provide crucial remarks such as routine screening of PTH in the subgroup of patients with ischemic stroke due to extracranial atherosclerosis.

The main limitation of our study was that we did not examine the subgroup of the hyperparathyroidism which is unable to suggest detailed discussion regarding the underlying pathophysiology of this association. For instance, we do not know if our patients compromised of primary or secondary hyperparathyroidism, the pathophysiologies, evaluation processes as well as treatments of which differ substantially. In addition, the variability as well as subgroups of primary hyperparathyroidism have been broadened in the recent reports, including symptomatic disease, asymptomatic variant, and normocalcemic primary hyperparathyroidism [25]. Therefore, based on our method, we cannot exclude the possible influence of other factors such as vitamin D level, serum calcium and phosphate level in the study results. For instance, hyperphosphatemia which is a cause of secondary hyperparathyroidism has been shown to be a driving force in the pathogenesis of vascular calcification [26]. However, the negative effect of PTH-related effects on vascular function has been shown in both primary hyperparathyroidism and secondary hyperparathyroidism [27]. Besides, there is a considerable amount of evidence supporting the role of solely PTH increment in the development of impaired vascular compliance, out of the effects of confounder factors (calcium, phosphate, vitamin D, etc.) [2]. However, we think that future reports of larger case series, whose subtypes of hyperparathyroidism would be detailed documented, are surely warranted to clarify these discussions.

\section{Conclusions}

In conclusion, herein, we found a high incidence of hyperparathyroidism in our group of patients with ischemic stroke. A remarkable result was that elevation of PTH was found to be significantly associated with the ischemic stroke subtype of extracranial atherosclerosis. Clarification of these results in the future large-scale studies may provide crucial perspectives regarding our understanding of the pathophysiology of some subtypes of ischemic stroke and potentially lead to a large public health implication in this area.

\section{Acknowledgments}

None to declare.

\section{Financial Disclosure}

The authors declared that this study has received no financial support.

\section{Conflict of Interest}

The authors have no conflict of interest to declare.

\section{Informed Consent}

The informed consent has been obtained from the participants.

\section{Author Contributions}

HO contributed to the concept, design, supervision, materials, data collection and/or processing, analysis and/or interpretation, literature search, writing of the manuscript, and critical review; GA contributed to the design, materials, data collection and/or processing, and writing of the manuscript.

\section{Data Availability}

The data supporting the findings of this study are available from the corresponding author upon reasonable request.

\section{References}

1. Vestergaard P, Mollerup CL, Frokjaer VG, Christiansen P, Blichert-Toft M, Mosekilde L. Cardiovascular events before and after surgery for primary hyperparathyroidism. World J Surg. 2003;27(2):216-222.

2. Walker MD, Fleischer J, Rundek T, McMahon DJ, Homma S, Sacco R, Silverberg SJ. Carotid vascular abnormalities in primary hyperparathyroidism. J Clin Endocrinol Metab. 2009;94(10):3849-3856.

3. Hagstrom E, Michaelsson K, Melhus H, Hansen T, Ahlstrom H, Johansson L, Ingelsson E, et al. Plasma-parathyroid hormone is associated with subclinical and clinical atherosclerotic disease in 2 community-based cohorts. Arterioscler Thromb Vasc Biol. 2014;34(7):1567-1573. 
4. Bostrom H, Alveryd A. Stroke in hyperparathyroidism. Acta Med Scand. 1972;192(4):299-308.

5. Sato Y, Kaji M, Metoki N, Satoh K, Iwamoto J. Does compensatory hyperparathyroidism predispose to ischemic stroke? Neurology. 2003;60(4):626-629.

6. Celik G, Dogan A, Dener S, Ozturk S, Kulaksizoglu S, Ekmekci H. Parathyroid Hormone Levels in the Prediction of Ischemic Stroke Risk. Dis Markers. 2017;2017:4343171.

7. Adams HP, Jr., Bendixen BH, Kappelle LJ, Biller J, Love BB, Gordon DL, Marsh EE, 3rd. Classification of subtype of acute ischemic stroke. Definitions for use in a multicenter clinical trial. TOAST. Trial of Org 10172 in Acute Stroke Treatment. Stroke. 1993;24(1):35-41.

8. Hedback G, Oden A, Tisell LE. The influence of surgery on the risk of death in patients with primary hyperparathyroidism. World J Surg. 1991;15(3):399-405; discussion 406-397.

9. Wu GY, Xu BD, Wu T, Wang XY, Wang TX, Zhang X, Wang $X$, et al. Correlation between serum parathyroid hormone levels and coronary artery calcification in patients without renal failure. Biomed Rep. 2016;5(5):601606.

10. Wermers RA, Khosla S, Atkinson EJ, Grant CS, Hodgson SF, O'Fallon WM, Melton LJ, 3rd. Survival after the diagnosis of hyperparathyroidism: a population-based study. Am J Med. 1998;104(2):115-122.

11. Schillaci G, Pucci G, Pirro M, Monacelli M, Scarponi AM, Manfredelli MR, Rondelli F, et al. Large-artery stiffness: a reversible marker of cardiovascular risk in primary hyperparathyroidism. Atherosclerosis. 2011;218(1):96101.

12. Goettsch C, Iwata H, Aikawa E. Parathyroid hormone: critical bridge between bone metabolism and cardiovascular disease. Arterioscler Thromb Vasc Biol. 2014;34(7):1333-1335.

13. Press DM, Siperstein AE, Berber E, Shin JJ, Metzger R, Monteiro R, Mino J, et al. The prevalence of undiagnosed and unrecognized primary hyperparathyroidism: a population-based analysis from the electronic medical record. Surgery. 2013;154(6):1232-1237; discussion 1237-1238.

14. Jorde R, Sundsfjord J, Haug E, Bonaa KH. Relation between low calcium intake, parathyroid hormone, and blood pressure. Hypertension. 2000;35(5):1154-1159.

15. Hagstrom E, Kilander L, Nylander R, Larsson EM, Michaelsson K, Melhus H, Ahlstrom H, et al. Plasma parathyroid hormone is associated with vascular demen- tia and cerebral hyperintensities in two community-based cohorts. J Clin Endocrinol Metab. 2014;99(11):41814189.

16. You R, McNeil JJ, O'Malley HM, Davis SM, Donnan GA. Risk factors for lacunar infarction syndromes. Neurology. 1995;45(8):1483-1487.

17. Wang Y, Xu J, Zhao X, Wang D, Wang C, Liu L, Wang A, et al. Association of hypertension with stroke recurrence depends on ischemic stroke subtype. Stroke. 2013;44(5):1232-1237.

18. Weder AB. Treating acute hypertension in the hospital: a Lacuna in the guidelines. Hypertension. 2011;57(1):1820.

19. Kim JS, Nah HW, Park SM, Kim SK, Cho KH, Lee J, Lee YS, et al. Risk factors and stroke mechanisms in atherosclerotic stroke: intracranial compared with extracranial and anterior compared with posterior circulation disease. Stroke. 2012;43(12):3313-3318.

20. Aboyans V, Lacroix P, Criqui MH. Large and small vessels atherosclerosis: similarities and differences. Prog Cardiovasc Dis. 2007;50(2):112-125.

21. Fisher CM. The arterial lesions underlying lacunes. Acta Neuropathol. 1968;12(1):1-15.

22. Saji N, Toba K, Sakurai T. Cerebral Small Vessel Disease and Arterial Stiffness: Tsunami Effect in the Brain? Pulse (Basel). 2016;3(3-4):182-189.

23. Willeit J, Kiechl S. Prevalence and risk factors of asymptomatic extracranial carotid artery atherosclerosis. A population-based study. Arterioscler Thromb. 1993;13(5):661-668.

24. Hagstrom E, Ahlstrom T, Arnlov J, Larsson A, Melhus $\mathrm{H}$, Hellman P, Lind L. Parathyroid hormone and calcium are independently associated with subclinical vascular disease in a community-based cohort. Atherosclerosis. 2015;238(2):420-426.

25. Bilezikian JP, Bandeira L, Khan A, Cusano NE. Hyperparathyroidism. Lancet. 2018;391(10116):168-178.

26. Block GA, Hulbert-Shearon TE, Levin NW, Port FK. Association of serum phosphorus and calcium $\mathrm{x}$ phosphate product with mortality risk in chronic hemodialysis patients: a national study. Am J Kidney Dis. 1998;31(4):607617.

27. Smith JC, Page MD, John R, Wheeler MH, Cockcroft JR, Scanlon MF, Davies JS. Augmentation of central arterial pressure in mild primary hyperparathyroidism. J Clin Endocrinol Metab. 2000;85(10):3515-3519. 